\title{
Giant aneurysm of the left main coronary artery in Takayasu aortitis
}

\author{
H Suzuki, H Daida, M Tanaka, H Sato, S Kawai, H Sakurai, H Yamaguchi
}

\begin{abstract}
Takayasu aortitis is a chronic inflammatory vasculitis characterised by stenosis or obliteration of large and medium sized arteries. Although coronary arteries are affected in approximately $10 \%$ of cases, most of the lesions are luminal narrowing, and coronary aneurysm formation is extremely rare. A case is described of giant aneurysm of the left main coronary artery complicated with Takayasu aortitis in a 46 year old Japanese woman who was followed until her death at age 71 . Pronounced intimal proliferation and adventitial fibrous thickening of the involved arterial wall usually induce constriction or occlusion at the orifice of the main branch of the aorta in Takayasu aortitis. However, systemic hypertension, which resulted from renovascular stenoses in this case, is likely to have enlarged the vessel lumen before replacement of medial and adventitial fibrosis after extensive destruction of medial elastic fibres in the left main coronary artery. Moreover, associations such as autoimmune hepatitis, chronic thyroiditis, and Sjögren syndrome strongly suggests that Takayasu aortitis may be an autoimmune disease. (Heart 1999;81:214-217)
\end{abstract}

Keywords: giant coronary aneurysm; Takayasu aortitis; renovascular hypertension; autoimmune disorders

Takayasu aortitis is a chronic inflammatory disease of arteries with unknown aetiology, which occurs predominantly in young women. It commonly involves elastic arteries such as the aorta and its main branches, and sometimes the pulmonary arteries. ${ }^{1-5}$ In addition to the common localisation of these vascular lesions, coronary angiographic and pathological studies have revealed coronary artery lesions in $9-11 \%$ of cases. ${ }^{6-8}$ Coronary artery involvement consists mostly of stenosis or occlusion of coronary ostia, which was first described by Frovig and Lokens ${ }^{9}$ as a fatal complication. Since then, coronary artery involvement has been reported mainly as occlusive change. Coronary aneurysms associated with Takayasu aortitis are extremely rare.

The aetiology of Takayasu aortitis is unknown, although clinical and experimental studies $^{2-5}$ 8-10 have been extensively performed since the first description by Takayasu in 1908. ${ }^{11}$ Several studies have suggested the participation of immunogenetic factors in the pathogenesis of this disease. ${ }^{8-10}$ Takayasu aortitis is known to occur in association with a number of systemic, non-specific, inflammatory diseases; rarely, rheumatoid arthritis, ${ }^{12}$ ankylosing spondylitis, ${ }^{13}$ ulcerative colitis, ${ }^{14}$ and autoimmune disorders ${ }^{10}$ such as systemic lupus erythematosus, polymyositis, and scleroderma. Such associations strongly suggest that Takayasu aortitis is an autoimmune disease.

We report a case of giant coronary artery aneurysm in Takayasu arteritis, associated with several autoimmune disorders (autoimmune hepatitis, Hashimoto thyroiditis, and Sjögren syndrome).

\section{Case report}

A 46 year old Japanese woman was admitted to our hospital because of occasional syncope. She had a 10 year history of chronic hepatitis of unknown cause. Her blood pressure was $170 / 60 \mathrm{~mm} \mathrm{Hg}$ in the left arm and 140/ $60 \mathrm{~mm} \mathrm{Hg}$ in the right arm. There was a III/IV systolic murmur along the right sternal border and a diastolic blowing murmur over the left.

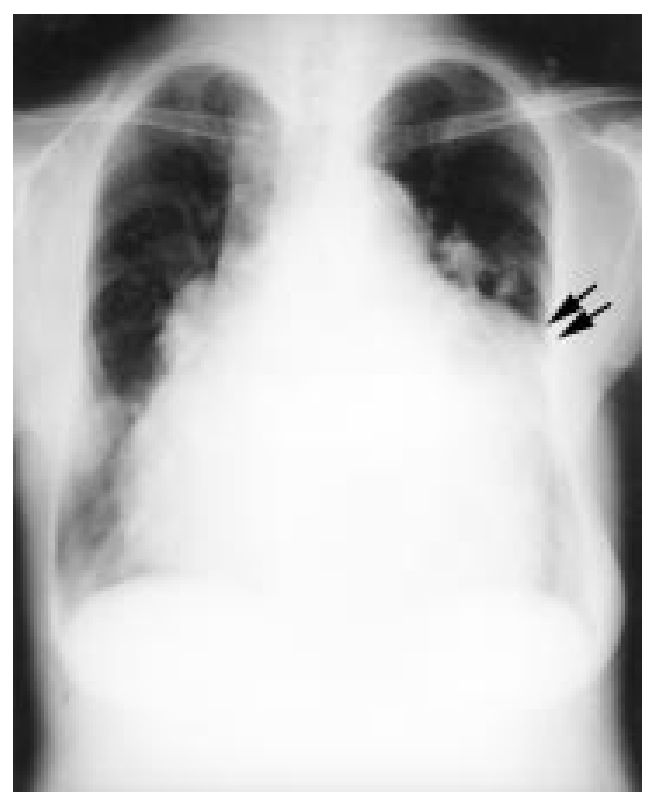

Figure 1 Severe cardiac enlargement and a prominent bulge (arrows) on the third arch of the left heart border. 


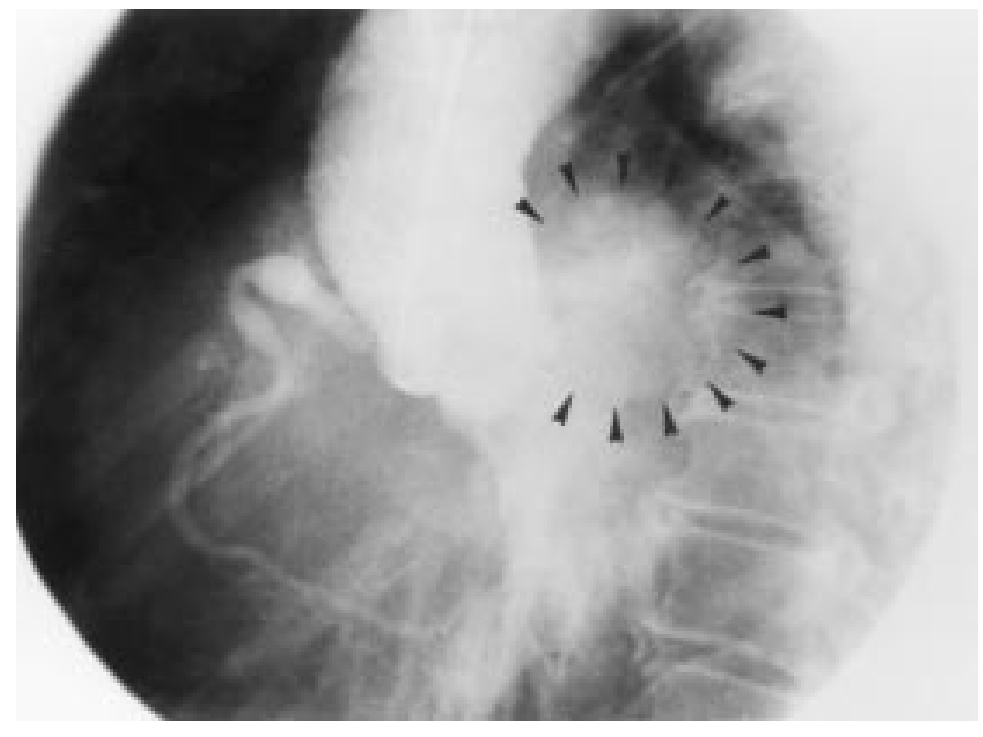

Figure 2 Left anterior cranial view of ascending aortogram at diastolic phase. A root aortogram shows dilatation of ascending aorta, severe aortic regurgitation, saccular or oval giant coronary aneurysm (surrounded by arrows) of left main stem, and small aneurysm of right coronary artery.

ECG disclosed atrial fibrillation and complete right bundle branch block. Laboratory studies gave either negative or normal results. There was no evidence of systemic inflammation: erythrocyte sedimentation rate was normal, C reactive protein was negative, and blood counts were normal. Additionally, serological test for syphilis, a test for rheumatoid factor, and lupus erythematosus preparations were negative, and an antinuclear antibody titre was $<1: 40$. Chest radiography showed severe cardiac enlargement and a prominent bulge on the third arch of the left heart border (fig 1). An aortogram showed severe aortic regurgitation, dilatation of the ascending aorta, and a localised narrowing of the abdominal aorta as well as severe calcification in the whole aorta (fig 2). She was diagnosed with healed aortitis and treated with digitalis and diuretics.

In 1986, at age 62, she was admitted again because of congestive heart failure. A selective coronary arteriogram revealed a saccular giant aneurysm of the left main coronary artery that was $5 \mathrm{~cm}$ in diameter without ostial stenosis, and a relatively small aneurysm of the proximal segment of the right coronary artery (fig 3). The left anterior descending and left circumflex arteries as well as the distal segment of right coronary artery were angiographically free from disease. Surgery was not undertaken because of severe calcification of the ascending aorta. Several antiplatelet and anticoagulant agents were administrated to prevent thromboembolism in the coronary aneurysm and its distal segment of coronary artery.

In January 1989, at age 65, she complained of general fatigue with massive pericardial effusion. She was diagnosed with primary hypothyroidism. Although she improved with thyroid hormone treatment, she often had congestive heart failure caused by severe aortic regurgitation and a progressive impairment of left ventricular contraction. In September 1995 , at age 71 , she died suddenly at home.

Postmortem examination revealed a saccular giant aneurysm of the left main coronary artery. The coronary aneurysm measured $5 \times 6 \times 5 \mathrm{~cm}$ without mural thrombus (fig 4). There was no ostial stenosis of the left main coronary artery. The coronary aneurysm had a fairly thickened wall and severe atherosclerotic changes. The right coronary artery also had a saccular aneurysm with a diameter of $2 \mathrm{~cm}$ at its proximal site. The whole aorta showed diffuse pronounced calcified arteriosclerosis, and a long segmental narrowing was observed in the abdominal aorta. The pulmonary arteries were severely dilated with atherosclerosis (diameter $3.5 \mathrm{~cm}$ ), and the orifices of the renal arteries were stenosed. However, muscular arteries such as the femoral arteries, coeliac artery, internal and external carotid arteries were not affected. In addition, there was root dilatation of the ascending aorta, causing the cusps failing to meet.

Microscopic examination showed extensive destruction of the media in the whole aorta and the proximal segments of the coronary arteries as well as the pulmonary arteries, which was replaced by dense hypocellular scarring fibrosis (fig 4) with severe fibrous thickening in the adventitia. Characteristic narrowing of vasa vasorum and onion skin-type fibromuscular thickening of their walls were noted in associ-
A

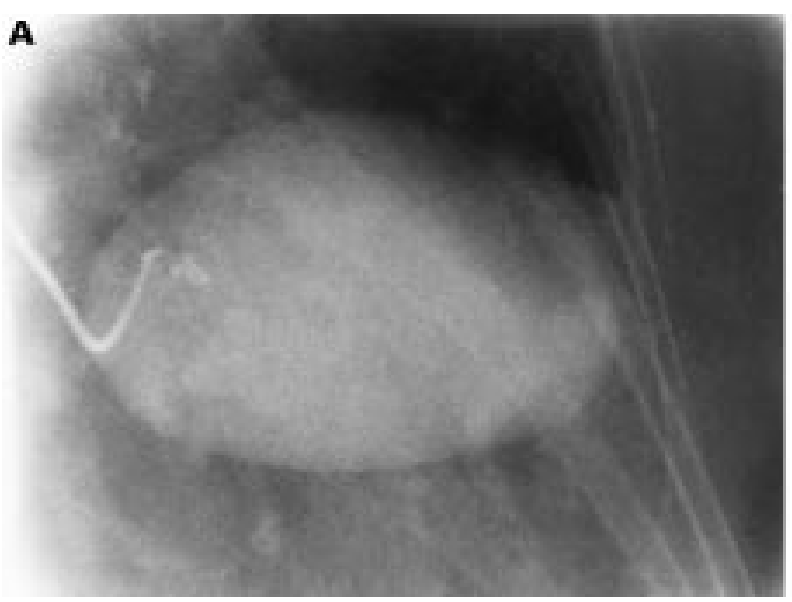

Figure 3 (A) Coronary angiogram showing saccular giant aneurysm of the left main coronary artery. (B) Coronary angiogram in the left anterior oblique showing an enlarged fusiform aneurysm in the proximal segment of the right coronary artery.

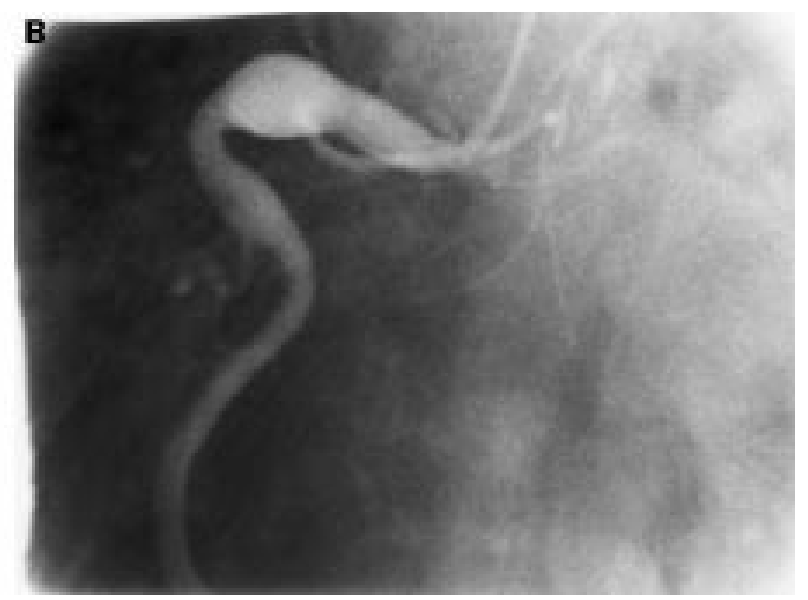



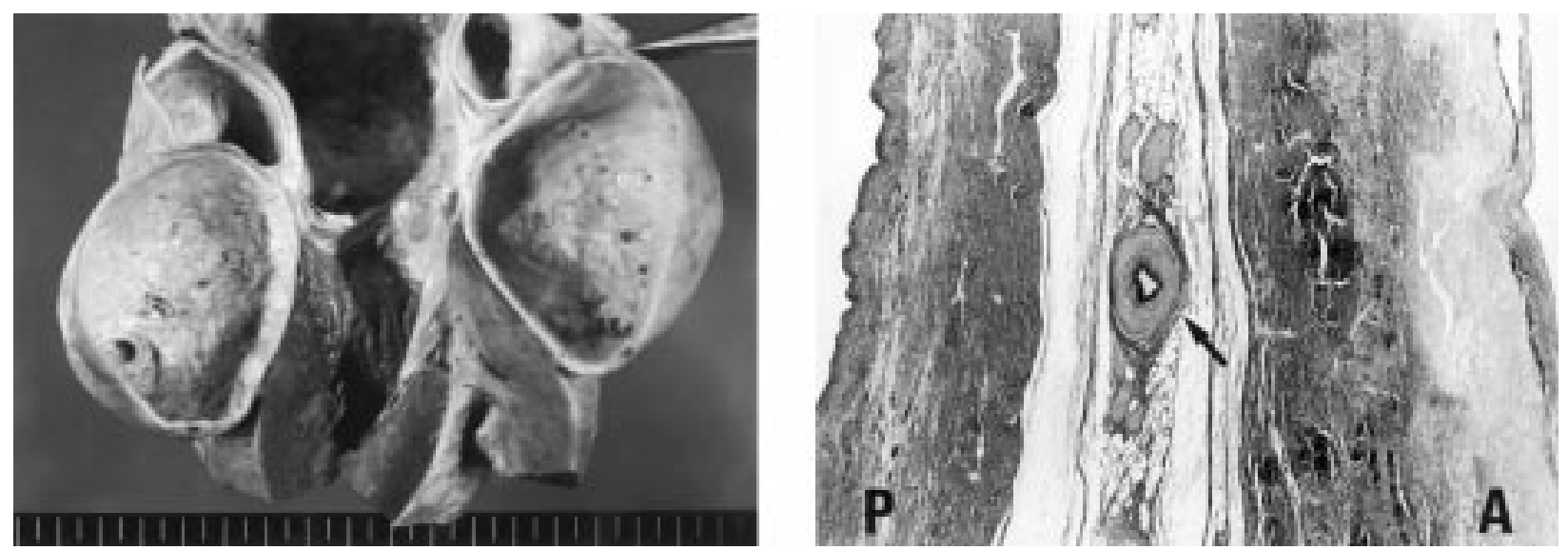

Figure 4 (Left) Massive aneurysm of left main coronary artery with its thickened wall and atherosclerotic change. (Right) Severely scarred media is surrounded by dense adventitial and atherosclerotic intima in the ascending aortic wall $(A)$ and the pulmonary arterial wall $(P)$. $A$ vasa vasorum (arrow) $i$ characteristically narrowed. (Elastic van Gieson stain, original magnification $\times 60$.)

ation with such adventitial changes. Histological features of the involved arteries were scar formation of media and adventitia in the healed stage of aortitis without the infiltration of leucocytes or giant cells.

Additional histological findings were chronic thyroiditis and sialoadenitis. Diffuse infiltration with lymphoid cells and plasma cells surrounding thyroid follicles was present. The thyroid follicles were small and the colloid sparse and densely stained. There were also periductal inflammatory infiltrates, fatty changes, and periductal fibrosis in submandibular glands.

\section{Discussion}

We know of only eight case reports of coronary aneurysm associated with Takayasu aortitis. ${ }^{5}{ }^{615-18}$ Lupi-Herrera et al described only one coronary aneurysm with fresh thrombosis in the right coronary artery found at necropsy in their series of Takayasu aortitis in which they reported the involvement of coronary arteries in 10 of 107 patients with aortitis syndrome. ${ }^{5}$ Rose et al also found coronary aneurysm formation at necropsy in two and total thrombotic occlusion with recanalisation in one of 16 patients with Takayasu aortitis. ${ }^{15}$

Severe intimal proliferation and adventitial fibrous thickening of the arterial wall usually induces constriction or occlusion at the orifice of the main branch of aorta, or coarctation of the abdominal aorta in Takayasu aortitis. In contrast, aneurysm formation such as dilatation of the ascending aorta seems to result from extensive destruction of elastic fibres in the media of the involved arterial wall as well as arterial hypertension, mainly from renovascular hypertension, which is most frequently found in patients with aortitis. ${ }^{1-5} 8-10$ Systemic hypertension is likely to accelerate atherosclerotic changes in major arteries and enlarge the vessel lumen of the involved arteries before massive replacement of medial and adventitial dense fibrosis, causing aneurysm formation.

A coronary aneurysm often causes stasis of blood flow and results in mural thrombosis and myocardial infarction; saccular aneurysms are thought to be frequently thrombosed, ruptured, or enlarged, resulting in myocardial in- farction, cardiac tamponade, or sudden death. ${ }^{13-15}$ Recent reports of surgical treatments for left main coronary arterial aneurysm encouraged coronary artery bypass operation with exclusion by ligation of the aneurysm to prevent these severe complications. ${ }^{18-20}$ Our patient had fairly long term survival with medical treatment without major cardiogenic complications, ${ }^{20}$ although the coronary arterial aneurysm of the left main coronary artery was saccular and very large. In our patient, both media and adventitia was replaced by pronounced fibrous thickening instead of the diffuse disruption of elastic layers in the media. The coronary aneurysm with its firm wall seems to have been protected from rupture; the wall of a coronary aneurysm associated with aortitis may be firmer than that complicated with atherosclerosis.

In conclusion, we report a case of Takayasu aortitis with giant aneurysm of the left main coronary artery that was followed up long term without surgical treatment. The case was characteristically associated with hepatitis, thyroiditis, and sialoadenitis. Further elucidation and follow up of many patients with aortitis are required to clarify its pathogenesis and to evaluate the best treatment.

1 Ueda H, Ito I, Okada R, et al. Aortic arch syndrome with special reference to pulseless disease and its variants. Fpn Heart $\mathcal{F} 1963 ; 4: 224-32$.

2 Nasu T. Pathology of pulseless disease: a systemic study and critical review of twenty-one autopsy cases reported in Japan. Angiology 1963;14:225-42.

3 Nakao K, Ikeda M, Kimata S, et al. Takayasu's arteritis: Nakao K, Ikeda M, Kimata S, et al. Takayasu's arteritis:
clinical report of eighty-four and immunological studies of clinical report of eighty-four and immunol

4 Committee report. Clinical and pathological studies of aortitis syndrome. Ipn Heart $\mathcal{f} 1968 ; 9: 76-87$.

5 Lupi-Herrera E, Sanchez-Torres G, Marcushamer J, et al. Lupi-Herrera E, Sanchez-Torres G, Marcushamer J, et al.
Takayasu's arteritis. Clinical study of 107 cases. Am Heart $\mathfrak{f}$ 1977;93:94-103.

6 Cipriano PR, Silverman JF, Perlroth MG, et al. Coronary arterial narrowing in Takayasu's aortitis. Am $\mathcal{f}$ Cardiol 1977;39:744-50.

7 Naoki M, Orita Y, Takeshita A, et al. Coronary arterial involvement in Takayasu's disease. Fpn Heart $\mathcal{F}$ 1981;23: 1007-13.

8 Nasu T. Takayasu's truncoarteritis; puseless disease or aortitis syndrome. Acta Pathol fpn 1982;32(suppl 1):117-31.

9 Frovig AG, Lokens AC. Syndrome of obliteration of the Frovig AG, Lokens AC. Syndrome of obliteration of the
arterial branches of the aortic arch due to aortitis. Acta Psyarterial branches of the aortic arch due
chiatr Neurol Scand 1951;26:313-37.

10 Ueda H, Saito Y, Ito I, et al. Immunological studies of aortitis syndrome. Fpn Heart $\mathcal{f} 1967 ; 8: 4-18$.

11 Takayasu M. A case with peculiar changes of the central retinal vessels in the retina. Acta Soc Ophthalmol fap 1908; 12:554-5. 
12 Rush PJ, Inman R, Reynolds WJ. Rheumatoid arthritis after Takayasu's arteritis. F Rheumatology 1986;13:427-30.

13 Paloheimo JA, Julkunen H, Siltanen P, et al. Takayasu's arteritis and ankylosing spondylitis. Acta Med Scand 1966; 179:77-85.

14 Achar KN, Al-Nakib B. Takayasu's arteritis and ulcerative colitis. Am f Gastroenterol 1986;81:1215-17.

15 Rose AG, Sinclair-Smith CC. Takayasu's arteritis. A study of 16 autopsy cases. Arch Pathol Lab Med 1980;104:231-7.

16 Matsubara O, Kuwata T, Nemoto T, et al. Coronary artery lesions in Takayasu arteritis: pathological considerations. Heart Vessels Suppl 1992;7:26-31.
17 Fukuhara M, Koyama O, Onishi K, et al. An autopsy case of aortitis syndrome with aneurysms of bilateral coronary arteries [in Japanese]. Shinzo (Heart) 1979;11:629-34.

18 Fukuda I, Iijima H, Itoh T, et al. Coronary artery aneurysm associated with aortitis syndrome diagnosed pre- and intraoperatively. Fpn Heart $\mathcal{F}$ 1983;24:1007-15

19 Fukaya Y, Miyakawa M, Senga O, et al. Surgical management of left main coronary artery aneurysm. Ann Thorac Surg 1994;57:228-30.

20 Ishikawa K, Maetani S. Long-term outcome for 120 Japanese patients with Takayasu's disease. Circulation 1994;90:1855-60

\section{A rare case of aortic valve endocarditis caused by Tropheryma whippelii with left coronary cusp perforation diagnosed by transoesophageal echocardiography and PCR}

Aortic valve endocarditis might be the first manifestation of Whipple's disease without further multiorgan involvement. A case is reported of aortic valve endocarditis with severe aortic regurgitation without clinical evidence of gastrointestinal disease in a 47 year old man who was admitted because of increasing dyspnoea and fatigue. During the patient's stay in hospital, infection parameters were rising while blood cultures remained negative. Multiplane three dimensional transoesophageal echocardiography showed increased end diastolic and end systolic diameters (72/ $57 \mathrm{~mm}$ ) with left ventricular dysfunction, enlarged atria, satellite vegetations on the anterior mitral valve leaflet and associated chordae, slight mitral regurgitation, and a flail aortic valve with vegetations on all coronary cusps, as well as a cusp perforation of the left coronary cusp, which was diagnosed by three dimensional reconstruction. The cusp perforation of the left coronary cusp is clearly visible as a black hole. The deformities caused by vegetations on the right coronary cusp (rc), noncoronary cusp (nc), and left coronary cusp (lc) are also evident (RA, right atrium; LA, left atrium; RCA, right coronary artery; LCA, left coronary artery). The diagnosis of aortic valve endocarditis caused by Tropheryma whippelii was made after aortic valve replacement, using

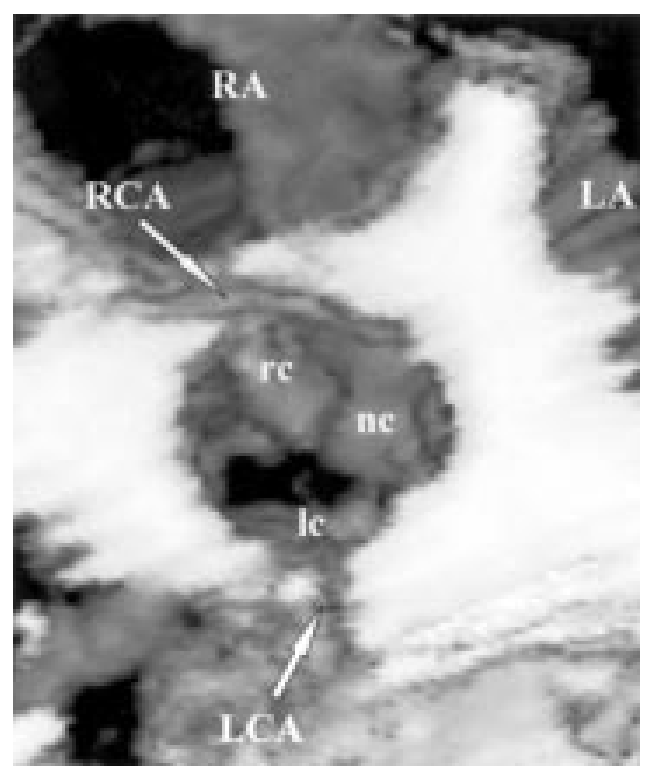

polymerase chain reaction of the aortic valve remnants and confirmed by DNA sequence analysis.

H F MANNAERTS

T HEKKER

C A VISSER 